\title{
MODELO CURRICULAR POR PROYECTOS: DISEÑO DE LOS CICLOS PROPEDÉUTICOS EN LA UNIVERSIDAD COLOMBIANA
}

\author{
Alhim Adonaí Vera Silva : Universidad Autónoma de Bucaramanga (Colombia) \\ avera6@unab.edu.co
}

\section{RESUMEN}

El modelo curricular por proyectos (MCP) que se propone para el diseño de los ciclos propedéuticos en la universidad colombiana ${ }^{2}$ podría sintetizarse en cuatro palabras: Necesidades, Problemas, Potencialidades y Proyectos. (NPPP). El modelo curricular tradicional se caracteriza por una agrupación, por lo regular aleatoria, de conocimientos de una disciplina, que a la vez puede subfragmentarse, con símbolos como: I, II, III, IV... estos fragmentos, trozos o pedazos, o asteroides de conocimientos flotan inconexos, desarticulados o abandonados en la mente del estudiante (memoria), esperando una oportunidad para demostrar su capacidad de resolver problemas complejos de sí mismo, la familia, la comunidad, la biodiversidad y del planeta interconectado en la globalidad.

\section{PALABRAS CLAVE: Modelo curricular, Propedéutico, Proyecto}

\section{ABSTRACT}

The curricular model for projects (CCM) proposed for the design of propaedeutic cycles in the Colombian university could be summarized in four words: Needs, Problems, Potentials and Projects. (NPPP). The traditional curricular model is characterized by a grouping, usually random, of knowledge of a discipline, which at

\footnotetext{
${ }^{1}$ Alhim Adonaí Vera Silva ${ }^{1}$ : Investigo, escribo y público libros académicos y guiones para módulos virtuales y presenciales derivados de los trabajos de campo en la educación desde preescolar hasta posgrados, en forma especial, en currículo, pedagogía, prospectiva y didácticas. He Par Académico Evaluador de careras universitarias; asesor y diseñador de currículos, modelos y módulos físicos y virtuales y he desarrollado estrategias didácticas. Representé a la Comunidad Académica de las universidades de Colombia ante el Consejo Nacional de Educación Superior (CESU) 1995 - 1997.

${ }^{2}$ http://www.mineducacion.gov.co/1621/article-196476.html Formación por ciclos propedéuticos, En Colombia predominaba el concepto de la Educación Técnica profesional y Tecnológica -TyT- como programas cerrados y concluyentes; es por eso que estos niveles de formación, los cuales hacen parte de la Educación Superior, no estaban articulados entre sí. Pero con la entrada en vigencia de la Ley 749 de 2002 esta realidad empezó a cambiar. Dicha ley introduce en el sistema educativo la formación por ciclos con carácter propedéutico, específicamente en las áreas de ingenierías, la tecnología de la información y la administración. Posteriormente, la Ley 1188 de 2008, la cual regula el registro calificado de programas de Educación Superior, amplía la posibilidad de formación por ciclos a todas las áreas del conocimiento. Según esta ley: "Todas las instituciones de Educación Superior podrán ofrecer programas académicos por ciclos propedéuticos hasta el nivel profesional en todos los campos y áreas del conocimiento dando cumplimiento a las condiciones de calidad previstas en la presente ley y ajustando las mismas a los diferentes niveles, modalidades y metodologías educativas".
} 
the same time can be subfragmented, with symbols such as: I, II, III, IV ... these fragments, pieces or pieces, or asteroids of Knowledge floats unconnected, disjointed or abandoned in the student's mind (memory), waiting for an opportunity to demonstrate its ability to solve complex problems of itself, the family, the community, biodiversity and the globally interconnected planet.

\section{KEY WORDS: Curriculum model, Propaedeutic, Project}

\section{Cómo citar el artículo:}

Alhim Adonaí Vera Silva. MODELO CURRICULAR POR PROYECTOS: DISEÑO DE LOS CICLOS PROPEDÉUTICOS EN LA UNIVERSIDAD COLOMBIANA. Revista de Ciencias de la Comunicación e Información. (Diciembre 2011). Año XXV (28), 17-36 ISSN: 0213-070-X

Recuperado:

http://www.revistaccinformacion.net/ARTICULOS/2011\%20Paula\%20Requeijo/2011\%20 Modelo\%20Curricular\%20por\%20Proyectos.\%20Vera\%20Alhim.pdf

\section{1.- Introducción:}

El modelo curricular por proyectos (MCP) tiende a acercar, el cerebro, el corazón y las manos; articular teoría - práctica, práctica - teoría, con visiones de una sociedad en red, interconectada por el conocimiento e Internet de las cosas con sensores. Establecer los puentes entre el conocimiento científico, tecnológico y artístico en la solución de los problemas concretos de la producción; la verificación en los campos teóricos de las apuestas conceptuales o las réplicas de experiencias significativas ${ }^{3}$ en el aprendizaje organizacional; la contratación de las innovaciones en varios escenarios de las empresas y de la sociedad; escrutar con mirada humanística los proyectos técnicos y tecnológicos que puede afectar de la fragilidad de los ecosistemas estratégicos y sus impactos en el calentamiento global del planeta; la investigación como eje central de la formación de los estudiantes a través de ciclos propedéuticos; el estudio de nuestras potencialidades en las materias primas, renovables y no renovables y sus sistemas de protección y explotación inteligente y con beneficio social; investigación que requiere alianzas con el sector productivo, regional e internacional, gran papel juegan los institutos y laboratorios de investigación en redes; así como el conocimiento generado en las prácticas de las cadenas productivas, como en el caso del Servicio Nacional de Aprendizaje SENA ${ }^{4}$, Colombia. El modelo curricular por proyectos (MCP) articula los semilleros de investigación con las redes fiscas y virtuales de cooperación científica regionales nacionales e internacionales.

Una de las características de una nueva organización del conocimiento consiste en la paulatina desaparición de las fronteras entre las teorías científicas que comienzan a materializarse en tecnologías y la integración de diversas tecnologías en una

\footnotetext{
3 http://www.oitcinterfor.org/sites/default/files/edit/docref/genero/siste expinn fautapo.pdf Universidad Amazónica de Pando, Bolivia. Guía para la Sistematización de Experiencias Innovadoras (2009).

${ }^{4}$ http://www.mineducacion.gov.co/cvn/1665/articles-246512 archivo pdf ErikaMoreno.pdf Servicio Nacional de Aprendizaje SENA, jóvenes rurales emprendedores, programa que construye competitividad y desarrollo en el campo colombiano, 2009, Colombia.
} 
lógica digital 'nano' que comienza a revolucionar los conocimientos científicos a través de la interdisciplinariedad, según puede significar pura y simplemente que diferentes disciplinas se coloquen en una misma mesa, en una misma asamblea, como las diferentes naciones se reúnen en la ONU sin poder hacer otra cosa que afirmar cada una sus propios derechos nacionales y su propia soberanía con respecto a las intromisiones del vecino... Pero, la interdisciplinariedad puede significar también intercambio y cooperación, lo cual hace que de ella resulte algo orgánico, la multidisciplinariedad y Transdisciplinariedad ${ }^{5}$ como su prefijo "trans" se interesa por lo que está a la vez entre las disciplinas, una forma de establecer puentes para resolver problemas, o anticipar situaciones críticas del planeta a través de las diferentes disciplinas. La metodología de la investigación transdisciplinar está determinada por los niveles de realidad lógica y la complejidad con un nuevo fenómeno de alianzas entre el sector productivo, empresarial, científico, tecnológico y político inédito para avanzar sobre la complejidad de las ciencias y sus fronteras difusas.

El modelo curricular por proyectos (MCP) estudia las megatendencias en la producción del conocimiento para anticipar los nuevos retos que el diseño curricular debe abordar desde los Proyectos Educativos Institucionales, se convierte en su baquiano, su guía, su camino para diseñar los perfiles, los conocimientos, contenidos-, las competencias, las organizaciones y las alianzas que los currículos exigen en la nueva universidad que algunos estudiosos como Axel Didriksson ${ }^{6}$ han venido llamando la Universidad de la Innovación en el siglo XXI. En este escenario de complejidad surgen nuevas lógicas en la relación ciudadano, biodiversidad, universo, familia, sociedad, producción que comienzan a cambiar conceptos alrededor de la vida, la salud, los alimentos, los servicios, la estética y la ética, entre otros incluso el surgimiento de conceptos como "ciudades educadoras" de las cuales hay experiencias significativas en Europa, fenómeno de una nueva arquitectura del conocimiento lo que abre escenarios de transformación de las cosmovisiones de ciudad, nación, planeta y universo y las formas como se abordan las ciencias, las tecnologías y las artes mediadas por Internet, en escenarios que permiten nuevos trabajos como la clonación, los transgénicos, desnudar los misterios del universo.

“¿Podrán los países Latinoamericanos con un enfoque del currículo tradicional asignaturista, formar por ciclos propedéuticos el talento humano que requiere una nación para analizar, investigar, innovar, y solucionar las necesidades y problemas de la sociedad $y$, dar valor agregado a las potencialidades con centros de investigación articulados al mundo productivo que generen nuevas empresas con nuestra materia primas, la diversidad cultural ambiental, mares, selvas, llanos y montañas, páramos y nevados; los conocimientos ancestrales, la multiculturalidad, el bienestar de la sociedad e impulso y transformación del universo de la producción de bienes tangibles e intangibles, en todos los escenarios con una distribución

\footnotetext{
${ }^{5}$ http://www.scielo.org.ve/scielo.php?pid=S0798-97922004000200007\&script=sci arttext $\quad$ Revista de Pedagogía, versión impresa ISSN 0798-9792, Rev. Ped v.25 n.73 Caracas mayo 2004, Transdisciplinariedad: Una propuesta para la Educación Superior en Venezuela, Miriam A. Carmona Rodríguez, Facultad de Ciencias y Centro de Estudios e Investigaciones sobre Educación Avanzada, Universidad Central de Venezuela, carmonam@rect.ucv.ve

${ }^{6}$ Maestro en estudios latinoamericanos y doctor en Economía. Fue director del Centro de estudios sobre la Universidad de la UNAM y Secretario de Educación del Departamento del Distrito Federal,
} 
equitativa y justa del bienestar de los ciudadanos de una nación?. La universidad permanece maniatada en una jaula de normas verticales de un Estado que teme perder el control de una universidad al vaivén de las megatendencias producidas por la globalización.

El modelo curricular por proyectos (MCP) toma los necesidades, problemas y potencialidades de la realidad en sus ámbitos planetarios, latino, nacional, regional e institucional y lo convierte en proyectos, con el propósito de formar talentos humanos en el campo técnico, tecnológico y profesional, donde la investigación se torna en estrategia para transformar la sociedad, con un valor agregado, que articula teoríapractica con impacto social, de tal forma que se tiende a sincronizar la situación problemática con la situación potenciadora dándole mayor riqueza a los proyectos como estrategia que, a la mediada por la investigación en los ciclos propedéuticos.

El (MCP) se centra en la dimensión pedagógica que propicia el desarrollo, el ejercicio de la autonomía de quien aprende, en la creatividad de quien construye los ambientes de aprendizaje, en la sociedad que recibe sus impactos y que se opone a la pasividad de quien aprende, a la verticalidad de quien "enseña". Es esencial precisar que el modelo busca ser un espacio para los trabajos en equipo, en redes físicas y virtuales-, con el compromiso de los actores en todas las fases de su proceso. Parte de la construcción colectiva del conocimiento desde distintos escenarios y desde diversos actores, veamos en forma breve las principales etapas del trabajo curricular por proyectos:

\section{Modelo curricular por proyectos (MCP)}

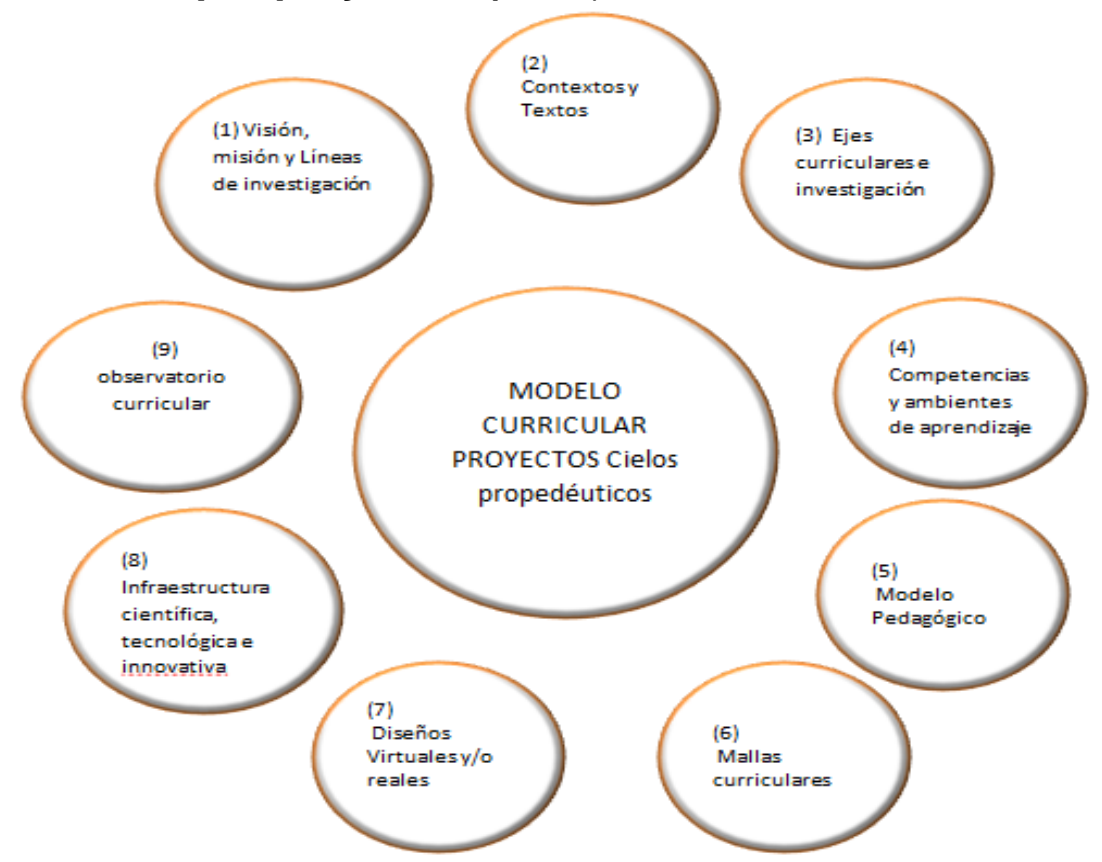

Fuente: Alhim Adonaí Vera Silva. Elaboración propia.

(1) Visión, misión y línea de investigación 


\section{MODELO CURRICULAR POR PROYECTOS: DISEÑO DE LOS CICLOS PROPEDÉUTICOS EN LA UNIVERSIDAD COLOMBIANA}

Las nueve fases del modelo curricular por proyectos (MCP) tienen como estrategia transversal los Talleres de Actores. El primer Taller de Actores, asume el estudio de megatendencias, los diagnósticos, las bases de datos, los estados del arte, las agendas prospectivas, el diseño de competencias y el diseño de ambientes virtuales o reales de aprendizaje, las mallas curriculares entre otros, que les permite al equipo de actores, el intercambio de conocimientos, frente a los futuros escenarios de la formación por ciclos propedéuticos. El taller de actores propicia los consensos y los disensos, al considerar la diferencia como uno de los elementos que enriquece la toma de decisiones, el intercambio de conocimientos con personas reconocidas por su talento en los sectores político, productivo, académico y social de los campos del conocimiento donde se pretende diseñar las carreras. Entre los actores encontramos: investigadores, educadores, estudiantes y personas con experiencias y conocimiento en el sector científico, tecnológico, académico, artístico, productivo, empresarial, gremial, laboral, político y social donde se desarrollaran los programas de formación universitaria por ciclos propedéuticos.

El taller de actores versa sobre las Megatendencias ${ }^{7}$ que es la dirección que toman simultáneamente varios aspectos de la sociedad (en términos tecnológicos, de nuevos productos, sistemas de producción y preferencias de consumo) y que tendrán un impacto cuyos efectos serán perceptibles por un segmento significativo de la sociedad por más de una década. En ciencia, tecnología e innovación de las carreras que se desea diseñar por ciclos propedéuticos. Este taller culmina con la formulación de la visión, la misión, frase sello y las líneas de investigación de las carreras, para lo cual se examinan los niveles de producción científica, innovación tecnológica, producción humanística, tanto en el ámbito global, en América Latina y el Caribe como en los contextos locales y se analizan las recomendaciones de los estudios prospectivos ${ }^{8}$ para constituye una anticipación para iluminar las acciones presentes con la luz de los futuros posibles y deseables para y de las agendas de ciencia y tecnología e innovación de COLCIENCIAS.

Cuadro guía para sistematizar la información solicitada por los actores

\begin{tabular}{|l|l|l|l|}
\hline \multicolumn{1}{|c|}{ Información } & \multicolumn{1}{|c|}{ Pasado } & Presente & Futuro \\
\hline $\begin{array}{l}\text { Megatendencias } \\
\text { planetarias en ciencia } \\
\text { tecnología e } \\
\text { innovaciones }\end{array}$ & & & \\
\hline Megatendencias & & & \\
\hline
\end{tabular}

\footnotetext{
${ }^{7}$ Las megatendencias tecnológicas actuales y su impacto de oportunidades estratégicas de negocios Icnológico de Monterrey (200). Título original. Compilado por: Grupo Coordinador de Desarrollo Regional del Tecnológico de Monterrey, Diseño de la publicación Adriana Hernández Márquez, Juan Carlos Muñoz Anaya. Primera edición, 2009. http://gestionestrategica.wikispaces.com/file/view/MegatendenciasTecnologicas y su ImpactoOportunidade sEstrategicas(1)+copia.pdf

${ }^{8}$ http://www.revistas.unal.edu.co/index.php/ingeinv/article/viewFile/21296/22265. Revista Ingeniería e Investigación No. 44 Diciembre de 1999 Metodología de los Escenarios para Estudios Prospectivos Alexandra v. Cely B. Ingeniería Química, Universidad Nacional de Colombia,
} 


\begin{tabular}{|l|l|l|l|}
\hline $\begin{array}{l}\text { Latinoaméricas en } \\
\text { ciencia tecnología e } \\
\text { innovaciones }\end{array}$ & & & \\
\hline $\begin{array}{l}\text { Megatendencias } \\
\text { Nacionales en ciencia } \\
\text { tecnología e } \\
\text { innovaciones }\end{array}$ & & & \\
\hline $\begin{array}{l}\text { Megatendencias } \\
\text { Regionales en ciencia } \\
\text { tecnología e } \\
\text { innovaciones }\end{array}$ & & & \\
\hline
\end{tabular}

El taller de actores estudia además las tendencias de las políticas internacionales, latinoamericanas nacionales y regionales sobre las carreras que se diseñan. Se examinan los planes de desarrollo, desde los globales, hasta los locales, e incluso institucionales, se valora los políticas de los organismos internacionales como la (Organización para la educación, la ciencia y la cultura de las Naciones Unidas) UNESCO. La ONU (Organismos integrado al sistema de las Naciones Unidas, la (Organización de los Estados Americanos), OEA, la (Organización para la cooperación y el Desarrollo Económicos) OCDE y la Banca Mundial. Se analizan las políticas de comunidades de naciones que se han asociado, como la comunidad Económica Europea, la liga de los Países Árabes, el grupo de países Asiáticos con el liderazgo del Japón, el mismo papel del coloso Chino en la nueva geografía mundial, los Tratado de Libre Comercio de América del Norte, EEUU, Canadá y el MERCOSUR, la (Comunidad Andina de Naciones) CAN. Se comparan los estudios diagnósticos elaborados por las distintas instituciones, los resultados de las mesas de concertación y los estudios de los centros provinciales que lidera el (Servicio Nacional de Aprendizaje) SENA. El taller de actores revisa los perfiles, competencias y currículos de las carreras similares existentes en diversos ámbitos con el propósito de contrastar, sus similitudes y diferencias.

Definidos los escenarios prospectivos, se precisa aún más, la formulación de la Visión, Misión y la frase sello. La Visión y Misión propician la concertación de la línea de investigación que sostiene la estructura curricular como generadora de conocimientos que da valor agregado producto de la investigación tecnológica, la innovación, las patentes y la producción de artículos y procesos dentro de las cadenas productoras de conocimiento.

Megatendencias en las políticas sobre las carreras que se van a diseñar

\begin{tabular}{|l|l|l|l|l|}
\hline \multicolumn{1}{|c|}{ Información } & Pasado & Presente & Futuro & Observaciones \\
\hline $\begin{array}{l}\text { Megatendencias } \\
\text { en las políticas } \\
\text { Planetarias }\end{array}$ & & & & \\
\hline $\begin{array}{l}\text { Megatendencias } \\
\text { en las políticas }\end{array}$ & & & & \\
\hline
\end{tabular}




\begin{tabular}{|l|l|l|l|l|}
\hline Latinoamérica & & & & \\
\hline $\begin{array}{l}\text { Megatendencias } \\
\text { en las políticas } \\
\text { Nacionales }\end{array}$ & & & & \\
\hline $\begin{array}{l}\text { Megatendencias } \\
\text { en las políticas } \\
\text { Regionales }\end{array}$ & & & & \\
\hline
\end{tabular}

Con base en los conocimientos desarrollados en las tendencias y políticas sobre la innovación en las carreras se van a diseñar los ciclos propedéuticos, lo que implica revisar la primera formulación de las Visiones y Misiones y ajustarlas a las nuevas condiciones. A título de guía se propone un cuadro que permite visualizar la coherencia entre la Visiones, (escenarios), Misiones (acciones) y Líneas de investigación de la Universidad.

Análisis de coherencias entre Visión, Misión y Líneas de investigación

\begin{tabular}{|l|l|l|l|}
\hline \multicolumn{1}{|c|}{ Información } & Visión & Misión & Líneas Investigación \\
\hline Universidad & & & \\
\hline Facultad & & & \\
\hline Carrera Profesional & & & \\
\hline Carrera Tecnológica & & & \\
\hline Carrera Técnica & & & \\
\hline
\end{tabular}

El Taller de Actores articula sus trabajos con las redes de cooperación científico tecnológicas en América Latina y el Caribe con el apoyo de redes académicas de alta velocidad, como la Red Nacional Académica de Tecnología Avanzada (RENATA); la red de Cooperación Latinoamericana de redes Académicas CLARA, la red GEANT, red Europea; la red Internet 3 EEUU; la red ALICE y sus nodos. EL seminario será la estrategia que sustenta la formación de jóvenes semilleros articulados con COLCIENCIAS a través de la línea de investigación que sustenta cada uno de los diseños curriculares.

La modernización en los diseños curriculares implica la modernización en la plataforma hardware de la universidad para avanzar sobre el sendero de la flexibilidad, interconectividad, accesibilidad, velocidad, instantaneidad e interfecundación de los conocimientos colocados en las redes Internet para los estudiantes. Esta modernización en la infraestructura fortalece la educación presencial y en forma progresiva abre espacios para fortalecer la educación virtual en próximos años. 
El trabajo de diseño curricular por ciclos, puede hacerse en forma descendente desde el nivel más avanzando doctoral de la formación académicas, hasta el técnico incluso la niñez o en forma ascendente, desde la niñez hasta el postdoctorado

(2) Contextos y textos (situación del problema actual, propuestas de solución a futuro, $y$ productos esperados)

El segundo taller de actores consiste en el análisis de contextos (realidad) y textos (documentos) (situación el problema actual, propuestas de solución a futuro, y productos esperados). Esta estrategia permite construir el futuro de las carreras que se concretan en los escenarios, a través de "Visiones" "futuribles" en palabras de Michel Godet y definir las "Misiones" a partir de las lecturas de textos y contextos, diagnósticos, y Estado del Arte, que lleven al estudiante a un aprendizaje significativo, a través de rutas donde debe primar la lúdica y arte, como el corazón de los currículos deseables, posibles y realizables de los programas a diseñar. En esencia en este taller de actores se cuenta con los conocimientos y los bancos de información actualizados que permiten tomar decisiones acertadas. La calidad de los conocimientos determina la calidad de las decisiones. Este ejercicio tiene que ver con el estudio de los tres momentos históricos, pasado, presente y futuro de las carreras a diseñar.

En los procesos tradicionales de diseño curricular, primero se elabora el diagnóstico y luego se formula la Visión y la Misión como una proyección lineal. En la prospectiva la visión y la misión orientan la sistematización de problemas, necesidades y potencialidades a partir de las visiones del futuro. Los conocimientos surgidos del taller de actores alimentan el banco de proyectos curriculares regionales construidos en la mesa de concertación entre el sector productivo, académicos, político, social y comienzan a darle cuerpo a las rutas de formación.

Para orientar el ejercicio se ha elaborado una guía síntesis que destaca los ámbitos planetario, latinoamericano, nacional y regional e incluso institucional, en la situación actual futura y los productos esperados. Las situaciones problemáticas tienden a variar dependiendo de la complejidad de los escenarios prospectados en las propuestas curriculares.

Síntesis de Textos y Contextos de la carreras "Situación problemática" a resolver, situación futura esperada, producción logradas sobre el campo de trabajo

\begin{tabular}{|l|l|l|l|}
\hline \multicolumn{1}{|c|}{ Información } & $\begin{array}{c}\text { Situación } \\
\text { problemática }\end{array}$ & Situación futura & $\begin{array}{c}\text { Producción esperada } \\
\text { (proyectos) }\end{array}$ \\
\hline Planetario & & & \\
\hline Latinoamericano & & & \\
\hline Nacional & & & \\
\hline Regional & & & \\
\hline
\end{tabular}


Es fundamental en cada momento precisar las principales necesidades, problemas, potencialidades, líneas de investigación y los proyectos que se aconseja presentar a través de gráficos o figuras, en lo ideal, tridimensionales. Esta síntesis tiene como propósito establecer las continuidades históricas de las necesidades, problemas y potencialidades y poder convertirlos en proyectos y líneas de investigación vistos a través de los ejes curriculares de las carreras que unen las fases de formación de los ciclos propedéuticos. A continuación encontrará una guía de la síntesis: Situación problémica, nombre del proyecto y perfiles académicos.

Síntesis: Situación problémica, nombre del proyecto y perfiles académicos

\begin{tabular}{|l|l|l|l|}
\hline \multicolumn{1}{|c|}{ Información } & Situación problémica & Nombre del proyecto & Perfiles académicos \\
\hline Planetario & & & \\
\hline Latinoamericano & & & \\
\hline Nacional & & & \\
\hline Regional & & & \\
\hline
\end{tabular}

El uso de gráficos y otros elementos visuales permiten una síntesis actual y prospectada de las necesidades, problemas, potencialidades, proyectos y perfiles.

(3) Definición de los Ejes curriculares o líneas de investigación de los proyectos

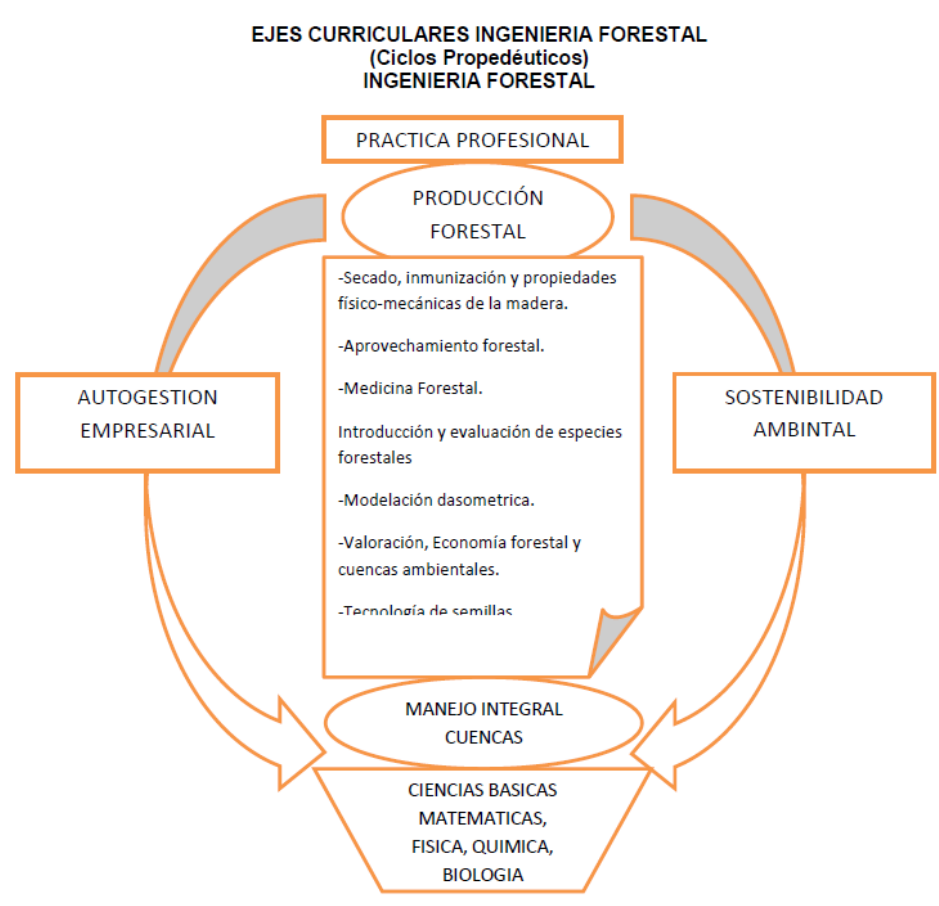

El tercer taller de actores se centra sobre la definición de los ejes curriculares y las líneas de investigación de los proyectos de cada una de las carreras que se diseñan. No se debe olvidar que los problemas, necesidades y potencialidades se transforman en proyectos que se materializan en ejes curriculares. Es esencial precisar que en el caso de las carreras técnicas, tecnológicas y profesionales por ciclos propedéuticos, existen ejes polivalentes transversal para las facultades de Ingeniería que les permite a los estudiantes que adquieran las competencias de este nivel acceder a cualquiera tecnología de ingeniería por ciclos propedéuticos basado en proyectos 0 en el campo de la administración 
organizacional, la gestión e interfecundación de conocimientos. Este eje común polivalente integrado por proyectos institucionales trasversales mediados por la investigación.

Veamos algunos ejemplos de simbolizaciones de carreras como Ingeniería Forestal, Ingeniería de Sistemas, Ingeniería Civil, y Administración Financiera con Énfasis Internacional.

Fuente: Alhim Adonaí Vera - Salomón Motta Manrique-Fanny Mercedes Gonzales Pinzón

Las necesidades potencialidades y problemas se transforman en proyectos que se materializan en ejes curriculares. En el caso de la ingeniería forestal surgen cuatro proyectos articulados en forma sistemática, que se convierten en ejes curriculares. Estos ejes son el resultado de la sistematización de necesidades, problemas y potencialidades que se abordarán desde la carrera en el desarrollo de competencias complejas: Manejo integral de cuencas hidrográficas; producción y posproducción forestal; autogestión empresarial, forestal y sostenibilidad ambiental en la producción y explotación forestal, cadenas productivas asociadas.

El principal eje de la formación es el proyecto de producciones y posproducciones forestal, que a su vez es la única línea de investigación "Producción y Posproducción Forestal con fines comerciales": Secado, inmunización de propiedades físicas de la madera aprovechamiento forestal, medición forestal, introducción y evaluación de especies forestales, modelación dasometrica, valoración, económica forestal, cuencas ambientales y tecnología de semillas.

El diseño simultáneo de varias carreras en el campo de la ingeniera, permite identificar proyectos ejes polivalentes transversales a las ingenieras como es el caso de la "Autogestión Empresarial" esencial en la formación de cualquier ingeniero. Este eje desborda el aprendizaje de competencias dirigidas al ejercicio de un empleo al adquirir competencias que le permite crear su propia empresa. 


\section{EJES CURRICULARES INGENERIA DE SISTEMAS \\ (Ciclos propedéuticos) \\ INGENIERIA DE SISTEMAS}

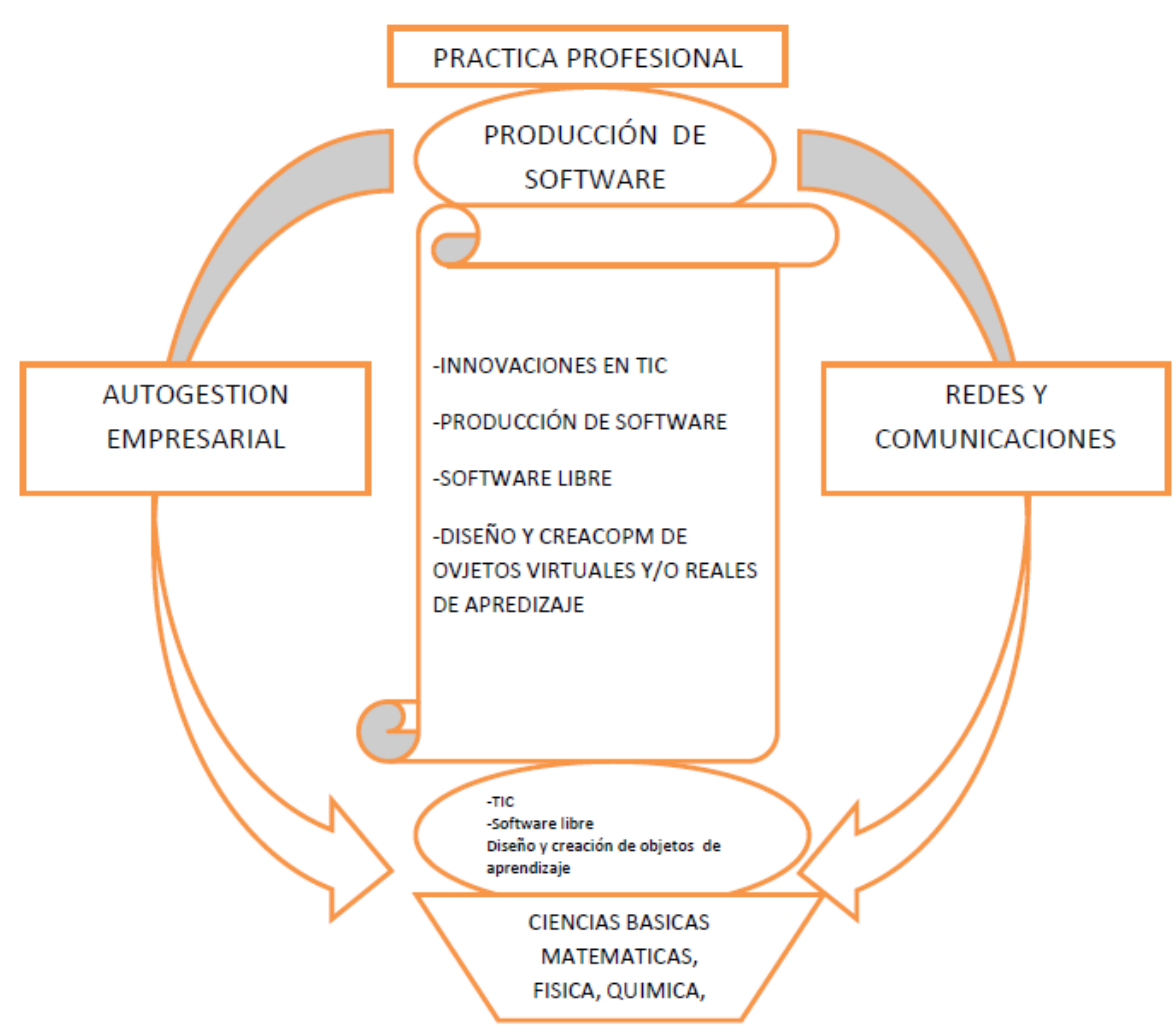

Fuente: Alhim Adonaí Vera - Henry Barón Zambrano, Fanny Mercedes González Pinzón 
Las potencialidades, problemas y necesidades simplemente se transforman en proyectos que se materializan en ejes curriculares. En el de caso de ingeniería de sistemas, surgen cuatro proyectos articulados en forma sistemática, que se convierten en ejes curriculares. Estos ejes proyectos son: Innovaciones Tecnologías (Diseño y creación de objetos virtuales y/o reales de aprendizaje); producción de software; Autogestión empresarial en Tecnología y Redes y comunicaciones. "Sistemas, Gestión del conocimiento y Redes;" herramienta fundamental del profesional que se comunica en forma instantánea con cualquier organización globalizado. El caso final de la identificación de un proyecto transversal polivalente lo constituye, el eje Biodiversidad y Sostenibilidad ambiental como una política de protección, preservación, explotación racional y reforestación permanente de nuestro hábitat natural planetario. A su vez, existen módulos presenciales o virtuales alrededor y manteniendo puentes conceptuales y experiencias significativas

que

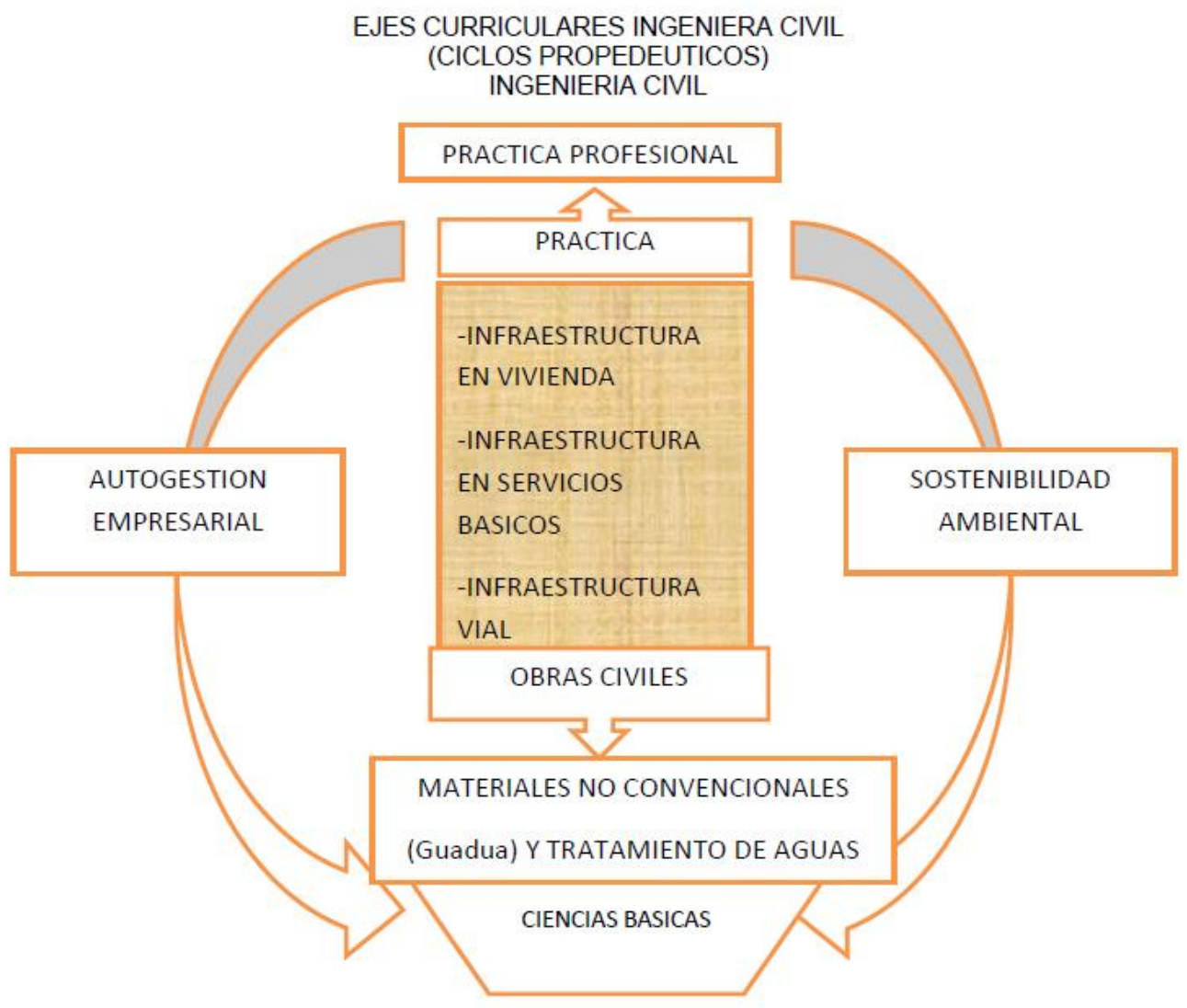

tributan a los proyectos.

Fuente:

Norma

Constanza

Guarnizo

Llanos,

Álvaro

Lozano

Osorio $y$

Alhim

Adonaí

Vera Silva

Las potenciales y problemas se transforman en proyectos que se materializan en ejes curriculares. En el caso de ingeniería Civil de las potencialidades, necesidades y potencialidades, surgen cuatro proyectos articulados en forma sistemática, que se convierten en ejes curriculares. Estos ejes del proyecto son pensados desde la prospectiva: infraestructura en vivienda, infraestructura en servicios básicos, infraestructural Vial, Autogestión Empresarial y sostenibilidad Ambiental. Los principales ejes son: Materiales no convencionales para construcción y Tratamiento de aguas como líneas de investigación. 
Para las carreras por ciclos ofrecidos en la modalidad virtual existen módulos introductorios, al conocimiento de las competencias, cuyo propósito radica en ofrecer el dominio de la comunicación interfecundación e innovación donde prevalezca los procesos de aprendizaje autónomo con ambientes que propicien el diseño, la simulación, la investigación y la innovación.

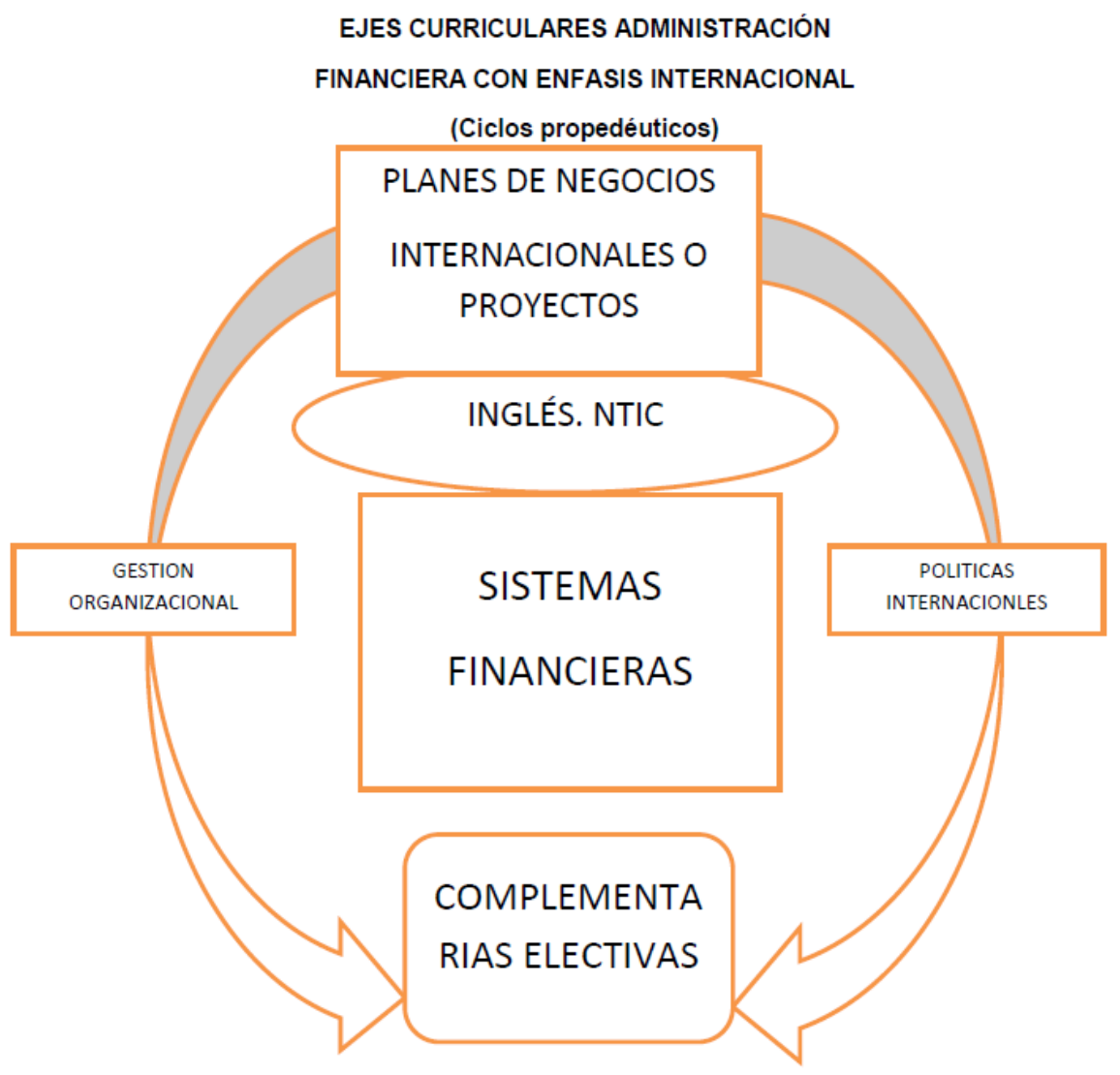

En la base de la gráfica se representan aquellas disciplinas que son necesarias para el desarrollo del proyecto programa Administración Financiera con Énfasis Internacional, como son las lógicas Matemáticas, Estadísticas y las Electivas de profundización. Posteriormente vienen los tres grandes ejes curriculares del programa: El eje central denominado Sistemas Financieros, que lo componen todas aquellas asignaturas como servicios, Financiero, Mercado de Capitales entre otras, propias del programa y que permiten obtener el conocimiento sobre el desarrollo de proyectos en el sector financiero nacional e internacional. Dos ejes paralelos proyectos, uno denominado proyectos de Gestión Organizacional que integra disciplinas como la administración, la Contabilidad, el Mercadeo y otras; el otro eje proyecto denominado Políticas Internacionales en donde se destaca toda la normatividad nacional e internacional, como Legislación Aduanera, Contratación Internacional, Relaciones Internacionales, Derecho Internacional, etc. El proyecto bilingüe y las nuevas tecnologías de la información son las disciplinas de apoyo del programa que facilitan la comunicación con el entorno internacional en el desarrollo de proyectos que impliquen el conocimiento de las culturas para lograr proyectos de 
gestión de recursos de capital especialmente para proyectos de inversión y/o planes de negocios a nivel global.

(4) Perfiles, Competencias, proyectos y ambientes de aprendizaje

El cuarto taller propicia la definición de perfiles, competencias, proyecto y ambientes de aprendizaje. Es la búsqueda de una solución inteligente al planteamiento de un problema tendiente a resolver, entre tantos, una necesidad humana, y por último los ambientes de aprendizaje son concebidos como construcción diaria, reflexión cotidiana, singularidad permanente que asegure la diversidad y con ella la riqueza de la vida. Los perfiles, las competencias, los proyectos, nutren los ambientes de aprendizaje. Para visualizar su articulación se ha diseñado un cuadro de doble entrada. En las columnas aparecen los perfiles, las competencias, los proyectos y los ambientes de aprendizaje y en las filas las competencias del ICFES relacionadas con el "ser", "hacer", "saber hacer", y "Saber convivir"9 Estas competencias siguen las categorías del modelo de pruebas ECAES del ICFES, descriptivas, argumentativas y propositivas. Pero el cuadro puede adaptarse a cualquier tipo de competencias.

Relaciones ente perfiles, competencias, proyectos y ambientes de aprendizaje

\begin{tabular}{|l|l|l|l|l|}
\hline \multicolumn{1}{|c|}{ Información } & Perfiles & Competencias & Proyectos & $\begin{array}{c}\text { Ambientes de } \\
\text { aprendizaje }\end{array}$ \\
\hline Ser & & & & \\
\hline Saber & & & & \\
\hline Hacer & & & & \\
\hline Convivir & & & & \\
\hline
\end{tabular}

Los perfiles, las competencias, los proyectos y los ambientes de aprendizaje se organizan con base en la priorización sustenta de los problemas, necesidades y potencialidades, así como de las características de la propuesta curricular. La investigación como eje curricular permanente es una de las principales innovaciones del modelo curricular por proyectos. Se podría decir, que la investigación es la cara de navegación del modelo porque a su interior se propician el desarrollo de las competencias científicas. Esta propuesta curricular busca nuevas formas de invención de ingenio de creatividad frente a la homogeneidad, a la rigidez y a la verticalidad. Se pretende construir una estrategia para darle estatuto a la flexibilidad, a la horizontalidad, a la cooperación y a la diferencia, en la educación del siglo XXI.

(5) Modelo pedagógico para los ciclos propedéuticos

\footnotetext{
9 Delors, J. (1996.): “Los cuatro pilares de la educación" en La educación encierra un tesoro. Informe a la UNESCO de la Comisión internacional sobre la educación para el siglo XXI, Madrid, España: Santillana/UNESCO. pp. 91-103.

Tomado de: $\underline{\text { http://uom.uib.es/digitalAssets/221/221918 9.pdf }}$
} 
El modelo se fundamenta en seis momentos relacionados con perfiles, competencias proyectos y ambientes de aprendizaje, experiencias significativas, conocimientos, redes virtuales mediados por la investigación y estrategias de evaluación centradas en el estudiante. La investigación al interior del modelo permite profundizar sobre el conocimiento construido por el hombre en las ciencias, las tecnologías y el arte con una visión humanística critica a través de la investigación, figura que se socializa, se multiplica y acompaña el proceso curricular desde el diseño hasta el la ejecución de las propuestas de carreras mediadas por proyectos de investigación, surgidos como se ha mencionada de manera reiterada de las necesidades, problemas y potencialidades. La investigación en una excelente estrategia para pasar de una educación centrada en la transmisión a una educación centrada en la investigación, para que los miembros del equipo de actores profundicen las relaciones entre el conocimiento científico, la innovación tecnología y la creación de empresas.

Boceto para construir un modelo pedagógico para los ciclos propedéuticos

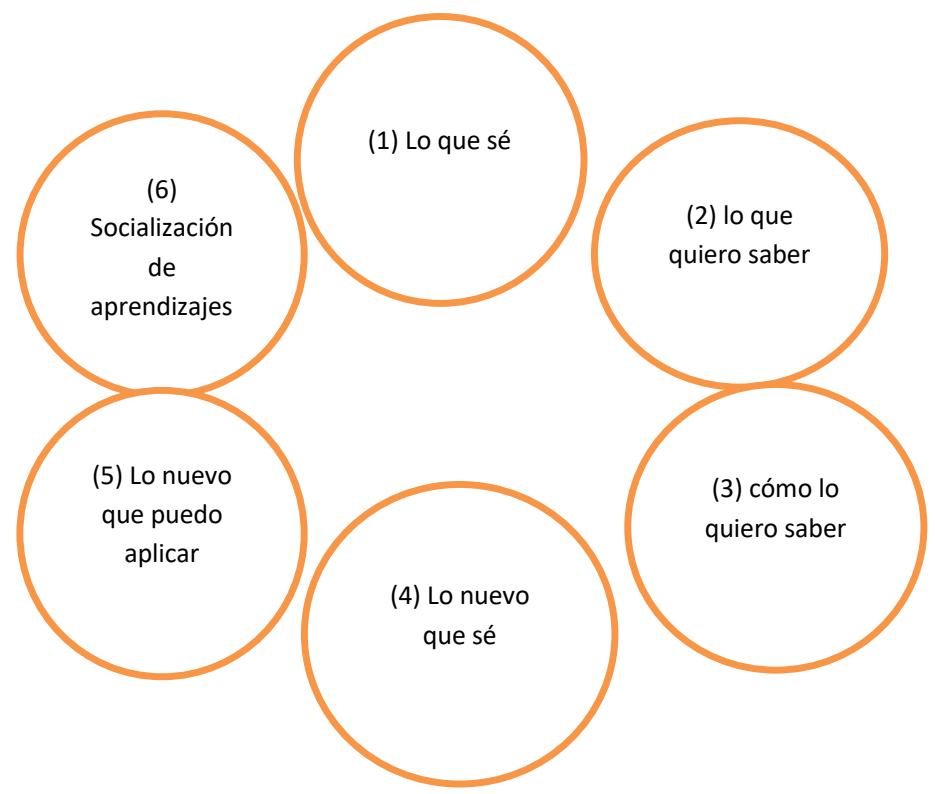

(6)

\section{Mallas curriculares en los ciclos propedéuticos}

El quinto taller de actores se relaciona con el diseño de las mallas curriculares dicho por (Rivera, 2009) es la estructura que da cuenta de la forma como los maestros abordan el conocimiento desde preescolar hasta undécimo grado. Es un instrumento que les permite, de manera comunitaria integrar las áreas desde diferentes enfoques, propiciando el diálogo entre saberes; es decir, una buena malla curricular conduce a los maestros a realizar su labor pedagógica articulada e integrada. Por lo tanto, la malla curricular proporciona una visión de conjunto sobre la estructura general de un área. Es un trabajo con esencia interdisciplinario donde los expertos tejen las relaciones entre la variadas competencias, conocimientos, ambientes de aprendizaje, metodologías, formas de evaluación y monitoreo previstas y las organizan en rutas abiertas tipo panal. Las mallas curriculares son una red de proyectos interconectados entre sí que permiten visualizar conexiones de aprendizaje. Las rutas son senderos del conocimiento. Formas de construir y 
reconstruir el conocimiento de las variadas disciplinas, que pueden proporcionar otras formaciones particulares para intereses particulares de los ciudadanos digitales. Las mallas curriculares les abren camino a profesores, estudiantes, investigadores, diseñadores y expertos para establecer las secuencias de los aprendizajes o precisar aún más, las características de los ambientes de aprendizaje donde se desenvuelven los proyectos.

En el diseño de las mallas curriculares la selección de las disciplinas, paradigmas, pre-paradigmas se hace desde la dimensión universal que las disciplinas poseen por la comunidad científica dentro del relativismo obvio de las ciencias. Allí se precisa la selección de las disciplinas, los conocimientos, los conceptos y los símbolos asociados o no, a los respetivos proyectos en su fase de ejecución. Este cruce de ejes: Eje diacrónico disciplinas, paradigmas, conceptos y símbolos, lo conceptual y el eje sincrónico lo contextual, orientado por los proyectos ejes curriculares permiten la relación entre teoría, práctica investigación e innovación que se sintetiza en la práctica empresarial que los estudiantes deben abordar como esencia de su formación profesional.

\section{(7) Diseño de ambientes virtuales o reales de aprendizaje en los ciclos propedéuticos}

El diseño de procesos virtuales o reales de aprendizaje es la organización interdisciplinaria de las secuencias, conexiones, equivalencia, polivalencias, puentes e intercambios de conocimientos y experiencias significativas acerca de un problema, proyecto, potencialidad, necesidad, disciplina, hipótesis, paradigma, teoría, concepto, invento e innovación, entre otras construcciones culturales del conocimiento racionada con el respectivo ciclo propedéutico.

El diseño de procesos virtuales, es una estrategia que desborda el diseño de objetos virtuales solitarios que luego se ensartan en un trabajo curricular descontextualizado. La estrategia de sistematizar y establecer puentes entre los módulos y los objetos virtuales de aprendizaje puede ser interesante cuando se organiza alrededor de unos problemas planetarios como el calentamiento global, los procesos productivos la redistribución y gestión del conocimiento, las nuevas lógicas de las organizaciones sociales o el colapso de las reservas hídricas globales. Estas rutas de módulos y objetos virtuales de aprendizaje prediseñados pueden ser organizados de forma panal e interconectados a las rutas curriculares, en ciclos propedéuticos relacionados con un tronco común como es el caso de las ingenierías, de la administración entre otras.

\section{Valoración de la infraestructura}

La valoración de la infraestructura física, tecnológica y artística permite verificar, valorar, precisar las necesidades del proyecto frente a las existencias institucionales. La valoración de la infraestructura es un proceso de garantía para la ejecución del proyecto y debe realizarse entre los distintos actores: de los sectores académico, político, productivo y social. Es una confrontación de inventarios entre lo que tenemos y debemos poseer para cumplir con la formación científica y tecnológica derivada de los diseños curriculares.

\section{(8) Monitoreo, auto-evaluación y acreditación}


El monitoreo, es el seguimiento rutinario de la información prioritaria de un programa, su progreso, sus actividades y sus resultados. La auto-evaluación es una actividad programada y sistemática de reflexión acerca de la propia acción desarrollada, sobre la base de información confiable, con la finalidad de emitir juicios valorativos fundamentados, consensuados y comunicables. Por último la acreditación deben incluir mecanismo flexibles de ajuste al diseño que implique la generación de nuevos modelos de gestión del conocimiento y de administración, más horizontales y participativos en relaciones de poder en la toma de decisiones; de ello depende el estatuto de legitimidad de las mismas propuestas curriculares, en tato que sean menos participativas tendrán menos compromisos los actores en su ejecución. El monitoreo está relacionado con los indicadores de calidad definidos a través las competencias, la infraestructura, el bienestar social de los estudiantes, las pruebas nacionales internacionales, la solidez humanística, científica, tecnológica, de los profesores, las publicaciones, las innovaciones las patentes, los prototipos, logrados a través de alianzas estratégicas internacionales y los trabajos en redes científicas relacionadas con las rutas curriculares de los ciclos propedéuticos.

Somos optimistas en América Latina y el Caribe, que con las alianzas internacionales y el compromiso como lberoamérica entre las universidades, crece el intercambio del conocimiento podemos abordare problemas, necesidades y potencialidades, comunes en nuestro destino de vida planetario, con procesos rigurosos de auto-evaluación, con la participación y concertación de los distintos actores del sector académico, científico, político, productivo y la misma sociedad civil donde se articulen la cultura desde lo local y el conocimiento desde lo universal, podemos abordar la complejidad del siglo XXI.

\section{Referencias}

Las megatendencias tecnológicas actuales y su impacto de oportunidades estratégicas de negocios Icnológico de Monterrey (200). Título original. Compilado por: Grupo Coordinador de Desarrollo Regional del Tecnológico de Monterrey, Diseño de la publicación Adriana Hernández Márquez, Juan Carlos Muñoz Anaya. Primera edición, 2009. Disponible en: http://gestionestrategica.wikispaces.com/file/view/MegatendenciasTecnologicas y su ImpactoOportunidadesEstrategicas(1)+copia.pdf

Formación por ciclos propedéuticos, En Colombia Ministerio de Educación Nacional de Colombia. Disponible en: http://www.mineducacion.gov.co/1621/article$\underline{196476 . h t m l}$

Servicio Nacional de Aprendizaje SENA, Jóvenes rurales emprendedores, programa que construye competitividad y desarrollo en el campo colombiano, 2009, Colombia. Disponible en: http://www.mineducacion.gov.co/cvn/1665/articles246512 archivo pdf ErikaMoreno.pdf

Universidad Amazónica de Pando, Bolivia. Guía para la Sistematización de Experiencias Innovadoras (2009) 
Disponible en:

http://www.oitcinterfor.org/sites/default/files/edit/docref/genero/siste expinn faut apo.pdf

Revista Ingeniería e Investigación No. 44 Diciembre de 1999 Metodología de los Escenarios para Estudios Prospectivos Alexandra v. Cely B. Ingeniería Química, Universidad Nacional de Colombia. Disponible en: http://www.revistas.unal.edu.co/index.php/ingeinv/article/viewFile/21296/22265

Revista de Pedagogía, versión impresa ISSN 0798-9792, Rev. Ped v.25 n.73 Caracas mayo 2004, Transdisciplinariedad: Una propuesta para la Educación Superior en Venezuela, Miriam A. Carmona Rodríguez, Facultad de Ciencias y Centro de Estudios e Investigaciones sobre Educación Avanzada, Universidad Central de Venezuela, carmonam@rect.ucv.ve Disponible en: http://www.scielo.org.ve/scielo.php?pid=S079897922004000200007\&script=sci arttext 